Research Paper

\title{
Risk Factors Analysis of Pathologically Confirmed Cervical Lymph Nodes Metastasis in Oral Squamous Cell Carcinoma Patients with Clinically Negative Cervical Lymph Node: Results from a Cancer Center of Central China
}

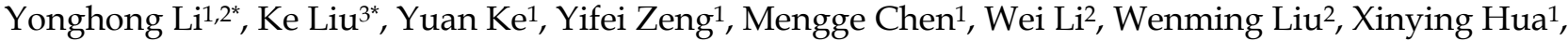 \\ Zheng $\mathrm{Li}^{1}$, Yahua Zhong ${ }^{1}$, Conghua Xie ${ }^{1}$, Haijun $\mathrm{Yu}^{1{ }^{1}}$
}

1. Department of Radiation and Medical Oncology, Zhongnan Hospital of Wuhan University; Hubei Cancer Clinical Study Center, Hubei Key Laboratory of Tumor Biological Behaviors, Wuhan, China

2. Department of Oncology and Surgery, The First Hospital of Tianmen City of Hubei Province, Tianmen, Hubei, China

3. Department of Oromaxillofacial and Head Neck Oncology, School and Hospital of Stomatology, Wuhan University, Wuhan, China

"Yonghong Li and Ke Liu contributed equally to this work

$\triangle$ Corresponding author: Haijun Yu, Mailing address: Department of Radiation and Medical Oncology, Zhongnan Hospital of Wuhan University, 169 Donghu Road, Wuhan 430000, China. E-mail: haijunyu@whu.edu.cn

(c) Ivyspring International Publisher. This is an open access article distributed under the terms of the Creative Commons Attribution (CC BY-NC) license (https://creativecommons.org/licenses/by-nc/4.0/). See http://ivyspring.com/terms for full terms and conditions.

Received: 2018.10.08; Accepted: 2019.04.27; Published: 2019.06.02

\begin{abstract}
Objective: To explore the risk factors of cervical lymph node metastasis in oral squamous cell carcinoma (OSCC) patients with clinical negative cervical lymph nodes $(\mathrm{cN} 0)$ and provide a reference for clinical treatment.

Methods: The clinical data of 161 OSCC patients with cN0 were retrospectively analyzed. All patients underwent extended primary resection combined with cervical lymph node dissection. The level and number of cervical lymph node metastasis were confirmed by postoperative pathology. The risk factors of cervical lymph node metastasis in patients were analyzed by univariate and multivariate Logistic regression analysis.

Results: Thirty-one out of 161 cases (19\%) were confirmed cervical lymph node metastasis. Among them, there were 28 cases of lymph node metastasis in one cervical level and 3 cases in two cervical levels. A total of 42 positive lymph nodes were detected in 34 cervical levels. The level number of positive areas in the IA, IB, IIA, IIB, III, IV and V levels was $2,15,12,1,4,0$, and 0 , respectively. The corresponding regional metastasis rates were $5.9 \%, 44.1 \%, 35.3 \%, 2.9 \%, 11.8 \%, 0 \%$ and $0 \%$, respectively. The number of positive lymph node metastases in the corresponding levels were $2,17,17,1,5,0$, and 0 respectively. Univariate analysis showed that gender, age, lesion location, T stage, and perineural invasion/lymphvascular invasion (PNI/PVI) had no significant effect on cervical lymph node metastasis $(P>0.05)$. The growth pattern, degree of differentiation, depth of invasion, neutrophil/lymphocyte ratio (NLR) and the short/long axis diameter ratio ( $\mathrm{S} / \mathrm{L}$ ratio) of lymph nodes were important factors influencing the cervical lymph node metastasis in $\mathrm{cNO}$ OSCC patients $(P<0.05)$. Multivariate Logistic regression analysis indicated that the growth pattern, degree of differentiation, depth of invasion, NLR, and the $S / L$ ratio of lymph nodes were independent risk factors for cervical lymph node metastasis $(P<0.05)$.

Conclusion: The growth pattern, degree of differentiation, depth of invasion, neutrophil/lymphocyte ratio, and the short/long axis diameter ratio of lymph nodes were the independent risk factors for pathological cervical lymph node metastasis in oral squamous cell carcinoma patients with cN0. If patients with the above risk factors receive nonstandard radical neck dissection or no dissection, it may be necessary for them to receive the corresponding regional postoperative radiotherapy.
\end{abstract}

Key words: Oral squamous cells carcinoma; lymph nodes metastasis; cervical lymph node dissection; risk factors 


\section{Introduction}

Oral squamous cell carcinoma (OSCC) is a common malignant tumor in the head and neck. Its incidence is $1.9 \% \sim 3.5 \%$ of the malignant tumor of the whole body, and it accounts for $4.7 \% \sim 20.3 \%$ of head and neck malignancies, ranking second in head and neck cancer[1-3]. Oral cancer can develop cervical lymph node metastasis early. Sometimes the lymph nodes are negative through clinical examinations such as palpation, B-ultrasonography, CT and MRI, but there are micrometastases in the lymph nodes after pathological examination after neck dissection, which is called occult lymphatic metastasis. About $20 \%$ to $40 \%$ of early stage oral cancer patients have occult cervical lymph node metastasis [4].OSCC is mainly metastasized through lymphatic channels. Related research [5-7] points out that once patients with oral squamous cell carcinoma are diagnosed with neck lymph node metastases, their survival rate can be significantly reduced. At present, there is no specific high-level early diagnosis for occult cervical lymph node metastasis in OSCC patients with cNO.

The rate of false negatives in routine palpation is relatively high. Imaging methods such as ultrasonography (US), magnetic resonance imaging (MRI) and computed tomography (CT) are usually applied to evaluate morphologic parameters such as nodal size, internal architecture and contrast enhancement pattern; but they cannot effectively detect micrometastasis in lymph nodes [8]. False positive/negative results of PET-CT also mislead the judgement of the cervical lymph nodes metastasis [9]. What's more, the expensive cost of PET-CT limits the regular use before operation in OSCC patients. Overtreatment or under treatment induced by the confusion of cN0 in OSCC patients will lead to treatment failure or complications of normal organs [10]. Patients who had no lymph node metastases (pN0) but underwent cervical lymph node dissection were subjected to unnecessary postoperative complications, such as intraoperative risk and shoulder dysfunction, clinical N0 patients with occult cervical lymph node metastasis but without cervical lymph node dissection will have the regional recurrence soon and bad prognosis. Therefore, the preoperative judgment of cervical lymph node metastases in cNO OSCC patients is a difficult and important point in clinical practice. Here we retrospectively analyzed the clinical data of OSCC patients with $\mathrm{cN} 0$ who underwent extended resection of the primary tumor combined with cervical lymph node dissection. We wanted to find the risk factors of cervical lymph node metastasis in OSCC patients with $\mathrm{cN} 0$ and provide references for surgical treatment and postoperative radiotherapy.

\section{Patients and Methods}

\section{Patients population}

This research has been approved by the Ethics Committee of Zhongnan Hospital of Wuhan University and Hospital of Stomatology of Wuhan University. All patients signed the informed consent before treatment. Inclusion criteria were following: (1) All patients were palpated by deputy director or chief surgeon, plain and contrast CT scans of the cervicofacial area were performed before surgery. No cervical lymph node enlargement was found in the cervical lymph nodes. The specific implementation criteria are: physical examination did not touch the enlarged lymph nodes, and the contrast CT scan of the head and neck did not show enlargement of the lymph nodes. The maximum diameter was less than 10 millimeters. There was no liquefaction or necrosis in the lymph nodes, and the peripheral annular reinforcement or irregular enhancement of the margins, disappearance of some or all of the peripheral fat space, or fusion of lymph nodes; (2) All patients underwent primary resection plus radical lymph node dissection or functional radical dissection; (3) All patients were confirmed as squamous cell carcinoma by postoperative pathological report. Postoperative diagnosis of cervical lymph nodes should be confirmed by pathology; (4) No radiotherapy or chemotherapy was performed before surgery. Exclusion criteria were following: (1) patients with incomplete data; (2) primary lesions located in other parts of the body; (3) patients diagnosed with cervical lymph node metastasis before surgery; (4) recurrence. Finally, 161 patients with oral squamous cell carcinoma who were admitted to the Hospital of Stomatology of Wuhan University from January 2014 to March 2017 were included in the study.

\section{Collecting of Clinical data}

A retrospective study was performed to record in detail the patient's gender, age, lesion location, $\mathrm{T}$ stage, growth pattern, differentiation, depth of invasion, NLR, the $\mathrm{S} / \mathrm{L}$ ratio of lymph nodes, PNI/PVI and other data. After detailed clinical examination after admission, all patients underwent extended resection of the primary tumor combined with cervical lymph node dissection. In our study, neck levels were recorded according to the classification proposed by the American Head and Neck Society. The neck lymph node pathological report was used as the gold standard. The detailed levels and numbers of positive metastasis cervical lymph node were recorded in the patients. 


\section{Statistical analysis}

All data were analyzed using SPSS 18.0 and Graphpad prism 7 software for statistical analysis. Count data are expressed as percentages (\%). The $\chi^{2}$ test was used for univariate analysis of the risk factors of cervical lymph node metastasis. Logistic regression analysis was applied for multivariate analysis of risk factors. The OR value was used to reflect the risk of cervical lymph node metastasis. $\mathrm{P}<0.05$ was considered as the difference with statistical significance.

\section{Results}

\section{Regional distribution and composition of cervical lymph node metastasis in OSCC patients with cNO}

In 161 OSCC patients with cN0, 2,415 lymph nodes were cleared during the operation with an average of approximately 15 nodes per patient. In our study, 31 out of 161 patients $(19 \%)$ presented with pathologically confirmed cervical lymph node metastases, including 1 case with pathologically lymph node micro-metastases. There were 28 cases of lymph node metastases in one cervical level and 3 cases of simultaneous lymph node metastases in two cervical levels. Two cases were IB and IIA, and one case was IIA and III. A total of 42 positive lymph nodes were detected in 34 cervical levels; the level number of positive nodes in IA, IB, IIA, IIB, III, IV, and $\mathrm{V}$ was $2,15,12,1,4,0$ and 0 , respectively. The corresponding metastasis rates of cervical levels were $5.9 \%, 44.1 \%, 35.3 \%, 2.9 \%, 11.8 \%, 0 \%$, and $0 \%$, respectively. The number of positive lymph nodes in the corresponding levels was 2, 17, 17, 1, 5, 0 and 0 , respectively. The detail data of positive lymph node levels and positive lymphatic nodes distribution are shown in Table 1.

Table 1. Pattern of distribution of cervical metastasis in the present series

\begin{tabular}{lll}
$\begin{array}{l}\text { Cervical } \\
\text { levels }\end{array}$ & $\begin{array}{l}\text { Number of metastasis levels } \\
(\%)\end{array}$ & $\begin{array}{l}\text { Number of metastasis nodes } \\
(\%)\end{array}$ \\
\hline Total (n) & 34 & 42 \\
Ia & $2(5.9 \%)$ & $2(4.8 \%)$ \\
Ib & $15(44.1 \%)$ & $17(40.5 \%)$ \\
IIa & $12(35.3 \%)$ & $17(40.5 \%)$ \\
IIb & $1(2.9 \%)$ & $1(2.3 \%)$ \\
III & $4(11.8 \%)$ & $5(11.9 \%)$ \\
IV & $0(0 \%)$ & $0(0 \%)$ \\
V & $0(0 \%)$ & $0(0 \%)$ \\
\hline
\end{tabular}

\section{Univariate analysis of risk factors of the cervical lymph node metastasis in OSCC patients with cNO}

Univariate analysis indicated that the depth of invasion, growth pattern, differentiation, NLR, the
S/L ratio and TNM postoperative stage were important risk factors in cervical nodal metastasis of OSCC patients with $\mathrm{cNO}$ but $\mathrm{pN}+$ after surgery $(P<0.05)$. Factors such as gender, age, lesion location, $\mathrm{T}$ stage and whether peripheral vascular/nerve invasion showed no effect on the final status of the cervical lymph node metastasis of the patients with $\mathrm{cN} 0$ in the present study $(P>0.05)$. The detailed results were demonstrated in Table 2.

Table 2. Univariate analysis of risk factors of the cervical lymph node metastasis in OSCC patients with cNO

\begin{tabular}{|c|c|c|c|c|c|}
\hline \multirow[t]{2}{*}{ Characteristics } & \multirow{2}{*}{$\begin{array}{l}\text { Case } \\
(n=161)\end{array}$} & \multicolumn{2}{|c|}{ Lymph node metastasis } & \multirow[t]{2}{*}{$x^{2}$} & \multirow[t]{2}{*}{$P$ Value } \\
\hline & & Yes (\%) & No $(\%)$ & & \\
\hline \multicolumn{6}{|l|}{ Gender } \\
\hline Male & 108 & $23(21.3)$ & $85(78.7)$ & 0.88 & 0.348 \\
\hline Female & 53 & $8(15.2)$ & $45(84.9)$ & & \\
\hline \multicolumn{6}{|l|}{ Age } \\
\hline$<60$ & 93 & $20(21.5)$ & $73(78.5)$ & 0.717 & 0.397 \\
\hline$\geq 60$ & 68 & $11(16.2)$ & $57(83.8)$ & & \\
\hline \multicolumn{6}{|l|}{ Primary site } \\
\hline Tongue & 87 & $16(18.4)$ & $71(81.6)$ & 1.632 & 0.897 \\
\hline Buccal mucosa & 38 & $9(23.7)$ & $29(76.3)$ & & \\
\hline Floor of mouth & 11 & $1(9.1)$ & $10(90.9)$ & & \\
\hline Gingiva & 13 & $2(15.4)$ & $11(84.6)$ & & \\
\hline Hard palate & 8 & $2(25)$ & $6(75)$ & & \\
\hline Retromolar & 4 & $1(25)$ & $3(75)$ & & \\
\hline \multicolumn{6}{|l|}{ T stage } \\
\hline T1 & 60 & $14(23.3)$ & $46(76.7)$ & 3.58 & 0.310 \\
\hline T2 & 77 & $11(14.3)$ & $66(85.7)$ & & \\
\hline T3 & 16 & $3(18.8)$ & $13(81.2)$ & & \\
\hline T4 & 8 & $3(37.5)$ & $5(62.5)$ & & \\
\hline \multicolumn{6}{|c|}{ Depth of invasion } \\
\hline$<4 \mathrm{~mm}$ & 128 & 20(15.6) & $108(84.4)$ & 5.292 & 0.021 \\
\hline$\geq 4 \mathrm{~mm}$ & 33 & $11(33.3)$ & $22(66.7)$ & & \\
\hline \multicolumn{6}{|l|}{ Growth type } \\
\hline Exogenesis & 39 & $10(25.6)$ & $29(74.4)$ & 11.853 & 0.003 \\
\hline Ulcerative & 93 & 10(10.7) & $83(89.3)$ & & \\
\hline Infiltrating & 29 & $11(37.9)$ & $18(62.1)$ & & \\
\hline \multicolumn{6}{|l|}{ Differentiation } \\
\hline Well & 77 & $10(13.0)$ & $67(87.0)$ & 14.893 & 0.001 \\
\hline Medium & 70 & $13(18.6)$ & $57(81.4)$ & & \\
\hline Poor & 14 & $8(57.1)$ & $6(42.9)$ & & \\
\hline \multicolumn{6}{|l|}{ NLR } \\
\hline$<3$ & 64 & $12(18.8)$ & $52(81.2)$ & 17.086 & 0.001 \\
\hline$\geq 3, \leq 3.99$ & 63 & $5(7.9)$ & $58(92.1)$ & & \\
\hline$\geq 4, \leq 4.99$ & 31 & $12(38.7)$ & $19(61.3)$ & & \\
\hline$>5$ & 3 & $2(67)$ & $1(33)$ & & \\
\hline \multicolumn{6}{|c|}{$\begin{array}{l}\text { Ratio of Short/long } \\
\text { axis diameter }\end{array}$} \\
\hline$<0.57$ & 124 & 19(15.3) & $105(84.7)$ & 10.764 & 0.005 \\
\hline$\geq 0.57, \leq 0.81$ & 31 & $8(25.8)$ & $23(74.2)$ & & \\
\hline$>0.81$ & 6 & $4(66.7)$ & $2(33.3)$ & & \\
\hline \multicolumn{6}{|c|}{$\begin{array}{l}\text { Perivascular or/and } \\
\text { perineural invasion }\end{array}$} \\
\hline No & 152 & $28(18.4)$ & $124(81.6)$ & 1.215 & 0.270 \\
\hline Yes & 9 & $3(33.3)$ & $6(66.7)$ & & \\
\hline \multicolumn{6}{|l|}{ TNM stage } \\
\hline I & 46 & $0(0)$ & $46(100)$ & 90.992 & 0.000 \\
\hline II & 67 & $0(0)$ & $67(100)$ & & \\
\hline III & 31 & $19(61.3)$ & $12(38.7)$ & & \\
\hline IVA & 17 & $12(70.6)$ & $5(29.4)$ & & \\
\hline
\end{tabular}

Multivariate logistic regression analysis of the risk factors of the cervical lymph node metastasis in patients with cNO

Multivariate Logistic regression analysis was performed on the depth of invasion, growth pattern, 
differentiation, NLR, the S/L ratio and TNM postoperative stage as independent variables. The results showed that the depth of invasion, growth pattern, differentiation, NLR, the S/L ratio and TNM postoperative stage were independent risk factors for cervical lymph node metastasis $(P<0.05)$. The detailed results are shown in the Table 3.

Table 3. Multivariate logistic regression analysis of the risk factors of the cervical lymph node metastasis in OSCC patients with $\mathrm{cNO}$

\begin{tabular}{llllll}
\hline Variables & $\begin{array}{l}\text { B } \\
\text { value }\end{array}$ & $\begin{array}{l}\text { S.E. } \\
\text { value }\end{array}$ & $\begin{array}{l}\text { Wald } \chi^{2} \\
\text { value }\end{array}$ & OR (95\% CI) & $\begin{array}{l}\boldsymbol{P} \\
\text { value }\end{array}$ \\
\hline $\begin{array}{l}\text { Depth of invasion } \\
\text { Growth type }\end{array}$ & 0.998 & 0.442 & 5.043 & $2.700(1.135-6.425)$ & 0.025 \\
Differentiation & 2.827 & 0.476 & 35.308 & $16.888(6.648-42.903)$ & 0.000 \\
NLR & 1.089 & 0.317 & 11.763 & $2.970(1.594-5.533)$ & 0.001 \\
$\begin{array}{l}\text { Ratio of short/long axis } \\
\text { diameter }\end{array}$ & 0.558 & 0.247 & 5.097 & $1.748(1.076-2.838)$ & 0.024 \\
TNM stage & 1.072 & 0.318 & 11.397 & $2.921(1.568-5.443)$ & 0.001 \\
& 2.641 & 0.458 & 33.195 & $14.026(5.712-34.443)$ & 0.000 \\
\hline
\end{tabular}

\section{Discussion}

Cervical lymph node metastasis is the main mode of metastasis in various stages of oral cancer, and it is also an important factor affecting the prognosis of oral cancer. The number and size of metastatic lymph nodes, whether or not the capsular invasion, and the extent of involvement of the neck region will directly affect the prognosis of patients [11]. Studies have shown that $50 \%$ of patients with oral cancer have lymph node metastasis at the first visit [12]. Researcher from Hunan Medical University analyzed 262 oral cancer patients and found that $5.3 \%$ patients had cervical lymph node metastasis as the first symptom [13]. Akhter $\mathrm{M}$ and colleagues found that the prognosis of patients with oral cancer with cervical lymph node metastasis is worse than that without lymph node metastasis, and the probability of distant metastasis increases when cervical lymph nodes metastasize [14]. Postoperative radiotherapy is the main treatment to reduce recurrence. Therefore, how to optimize the radiotherapy target area and accurately give radiotherapy to tumor tissue, and to protect normal tissue as much as possible is a particularly important content in the current radiotherapy target area delineation. An important prerequisite for optimizing the target area is to understand the relevant laws of cervical lymph node metastasis. The main purpose of this study was to explore the nature of lymph node metastasis and provide reference for postoperative radiotherapy.

Clinically, there is controversy over the management of cervical lymph nodes for patients with early cN0 oral squamous cell carcinoma. Some researchers advocate the policy of "wait-and-see". The "wait-and-see" strategy suggests that lymph node dissection is performed when obvious lymph node metastases are confirmed, but this treatment program significantly reduces the survival rate of the patients. The extended primary tumor resection combined with cervical lymph node dissection has a high cure rate. However, another believe that it has expanded the scope of surgery, causing shape damage and loss of some of the early functions of patients, affecting patients' quality of life [15]. Therefore, how to effectively evaluate cervical lymph node-negative patients with non-invasive cervical lymph node metastasis or high-risk metastases has always been the focus of clinical research.

The results of this study found that growth pattern, differentiation degree, depth of invasion, neutrophil/lymphocyte ratio, and ratio of short to long diameter of lymph nodes are important factors affecting the cervical lymph node metastasis of oral squamous cell carcinoma of cN0; and further analysis found that the growth pattern, degree of differentiation, depth of invasion, neutrophil/ lymphocyte ratio, and ratio of lymph node length to diameter were all independent risk factors for cervical lymph node metastasis.

Firstly, T stage basically represents the size of the tumor. The larger the tumor diameter, the higher the risk of lymph node metastasis. The gradual increase in tumor diameter is a result of the progression of the disease. The measurement of tumor diameter can reflect the approximate extent of the primary tumor, and when the diameter is large, it is easy to invade the surrounding tissue structure. In general, we believe that the larger the diameter of the tumor, the more likely it is that lymph node metastasis occurs in the neck. A number of studies have described the relationship between tumor size and lymph node metastasis. It is generally believed that the later the $\mathrm{T}$ stage, the higher the risk of lymph node metastasis. A study of Monroe MM et al [16] from the Oxford University Evidence-Based Medicine Center found that patients with oral CNN stage $\mathrm{T} 1$ have an occult metastasis rate of $18 \%$ to $30 \%$, and the chance of occult metastases in T2 patients rises to $24 \%-53 \%$. Koo et al [17] reported a retrospective analysis of 66 patients with stage N0-2 oral cancer and revealed that the rate of recessive metastasis was $8 \%$ in patients with $\mathrm{T} 2$, $25 \%$ in $\mathrm{T} 3,18 \%$ in $\mathrm{T} 4$, and no metastasis in $\mathrm{T} 1$. The lymph node metastasis rate from different studies may be different, but overall, the later the stage, the higher the risk of lymph node metastasis. In the present study, lymph node metastasis occurred in $30.4 \%$ of the $\mathrm{T} 1$ group, $16.6 \%$ in the $\mathrm{T} 2$ group, $23 \%$ in the T3 group, and $60 \%$ in the T4 group. Unfortunately, there is no statistical difference between $\mathrm{T}$ stage and lymph node metastasis in our study. However, we found that the rate of lymph node metastasis in the T4 
group is as high as $60 \%$, which is comparable with the results of most studies [18-20]. Therefore, for patients with cervical lymph node negative oral squamous cell carcinoma, if the $\mathrm{T}$ stage is late, cervical lymph node dissection should be performed on the basis of resection of the lesion in order to improve the cure rate.

The depth of invasion of oral squamous cell carcinoma affects the prognosis of patients. A large amount of literature [21] has reported to confirm that the depth of tumor infiltration was significantly correlated with cervical lymph node metastasis. In multivariate analysis, tumor infiltration depth is an independent prognostic factor and associated with tumor size, especially for the patients with T1-T2 stage. Kane SV et al [22] retrospectively studied 48 patients with early oral cancer who had undergone primary tumor resection and selective cervical lymph node dissection. The relationship between $\mathrm{T}$ size, tumor depth and thickness, degree of differentiation, invasion patterns, inflammatory response, unnaturalness, and lymph vascular infiltration and cervical lymph node metastasis was analyzed. Logistic regression analysis was used to determine the statistical significance of various parameters as predictors of subclinical node metastases. Fukano $\mathrm{H}$ et al [23] believed that the depth of invasion is the most important predictor of early oral squamous cell carcinoma. Pentenero et al [6] reviewed 55 articles with nearly 6,000 oral cancer patients with different clinical stages, sites and different methods of measurement in different studies. The threshold of infiltration depth varied from 1.5 to $10 \mathrm{~mm}$ in different studies, and the mode and median values were both at $4 \mathrm{~mm}$. The infiltration depth threshold of $4 \mathrm{~mm}$ was accepted as the criteria to determine whether or not the patients with cN0 undergo selective neck treatment. Melchers et al [24] obtained a threshold of invasive depth of $4.59 \mathrm{~mm}$ by ROC curve analysis, in which the infiltration depth of $4 \mathrm{~mm}$ can be used as a critical value for judging cervical lymph node metastasis in early oral cancer. Our study also came to the same result by univariate and multivariate analysis. Patients with a tumor infiltration depth greater than or equal to $4 \mathrm{~mm}$ have an increased risk of lymph node metastasis, radical or elective neck dissection for initial therapy or post-operative radiotherapy without neck dissection is highly recommended.

The growth pattern of oral squamous cell carcinoma is also one of the important factors that affect the prognosis of patients. According to the different growth modes of oral squamous cell carcinoma, it can be divided into exogenous type, ulcer type, and infiltrating type. Among them, exogenous type mainly presents rapid proliferation to the skin surface, and forms cauliflower with multiple infection or necrosis. Infiltration of cancer cells into the deep and peripheral lymph nodes invasion is not obvious for exogenous type, and therefore the rate of cervical lymph node metastasis is low. Ulcerative oral squamous cell carcinoma is more often found in the surface of the skin or mucosa with less infiltration to the deep, so the lymph node metastasis rate is also lower. The invasive oral squamous cell carcinoma has a rapid growth rate with early invading the surrounding tissues such as muscle and submucosa and early infiltration into the deep. Therefore, the lymph node metastasis rate of the neck is significantly higher than that of other types [25]. In this study, the positive rate of lymph node metastasis in oral cancer patients with invasive type was the highest (37.9\%), while that of exogenous and ulcer types was $25.4 \%$ and $10.7 \%$, respectively. There was a significant correlation between the growth pattern and the lymph nodes metastasis by the univariate Logistic regression analysis in the present study. (OR value of the growth pattern: 1.795). So we believe that there is high risk of the cervical lymph nodes metastasis for the oral squamous cells cancer patients with the invasive growth pattern even if the cervical lymph nodes are negative The degree of differentiation of oral squamous cell carcinoma reflects the degree of malignancy of oral squamous cell carcinoma. Haksever et al [26] pointed out that cervical lymph node metastasis in patients with oral squamous cell carcinoma is closely related to the degree of tumor differentiation, and as the degree of tumor differentiation decreases, the rate of cervical lymph node metastasis gradually increases. Other relevant studies [27] have also reported that the rate of cervical lymph node metastasis was only $9 \%$ in patients with well-differentiated oral squamous cell carcinoma, with $50 \%$ and $53 \%$ in moderately differentiated and poorly differentiated patients. In this study, the rate of positive lymph node metastasis in poorly differentiated oral squamous cell carcinoma was $57.1 \%$, which is much higher than that in well-differentiated oral squamous cell carcinoma $(12.9 \%)$ and moderately differentiated oral squamous cell carcinoma $(18.6 \%)$. There is a significant correlation between the degree of differentiation and the lymph nodes metastasis by the univariate Logistic regression analysis $(\mathrm{OR}=2.97)$. Therefore, it is recommended to perform cervical lymph node dissection for moderately and poorly differentiated patients at the same time.

NLR is recognized as a newly discovered tumor prognosis related indicator, more and more attention has been paid to it. Increased NLR suggests systemic 
inflammatory conditions that may present increased neutrophilia, decreased lymphopenia, or a combination of both. It is associated with disease states including endometriosis, acute coronary syndrome, Alzheimer's disease, and various cancers [28]. Hikaru Nakashima [29] measured the NLR in 124 patients with oral cancer prior treatment and analyzed the correlation between the NLR and various clinic pathological characterizes, they found that the elevated NLR was significantly associated with T3-4 stage and side effects of radio-chemotherapy. Saurabh Bobdey et al [30] retrospectively analyzed the medical records of 471 patients with oral cancer diagnosed between January 2007 and December 2008. Curve analysis and Cox proportional hazards analysis were used to evaluate the correlation between white blood cell count and overall survival. It was found that elevated monocyte counts (NLR> 2.38) were associated with poor overall survival (OS). Multivariate Cox proportional hazards analysis showed that high monocyte and NLR levels are important independent predictors of poor prognosis OS. It is suggested that NLR may be a potential biomarker that can predict the survival of clinical response to radiotherapy and chemotherapy. The enhanced systemic inflammatory response may be a potential target for improving patient prognosis. We divided the NLR ratios into 4 groups, $=<3,3-3.99$, $4-4.99,>=5$ in the present study. Univariate and multivariate logistic regression analysis revealed that there were significant differences of the lymph node metastasis rate among them. There is a trend that the lymph node metastasis rate is increasing depending on the NLR. Furthermore, the NLR can be obtained multiple times in preoperative laboratory tests and is a very convenient prognostic indicator with important guiding value.

The short to long axis diameter ratio(S/L ratio) of lymph nodes in tumor patients has been a concerned index of prognosis for clinicians [31]. Many people have received timely treatment because of early detection of abnormalities in short to long axis diameter ratio of lymph nodes in oral cancer patients. Stein kamp HJ [31] investigated 730 cervical lymph nodes in 285 patients and found that the nodes presenting with a more circular shape and an S/L ratio of more than 0.5 were diagnosed correctly as metastases with 95\% accuracy. Chi et al [32] retrospectively review 153 treated head and neck cancer patients with cervical lymph nodes who had received US and ultrasound -guided fine needle aspiration (FNA) and core needle biopsy, they found that the $\mathrm{S} / \mathrm{L}$ axis ratio $>0.5$ is an effective future in predicting the recurrence Lymph nodes in the treated neck. Hyung Won Lee [33] grouped the lymph nodes of breast cancer patients into benign and malignant groups, the ratio of short to long diameter of the benign group was $0.45 \pm 0.10$ and that of the malignant group was $0.59 \pm 0.17$, and the difference between them was significant $(\mathrm{P}=0.041)$. Therefore, we believe that the critical value of the S/L ratio of cervical lymph nodes is not yet clear and needs further investigation. In our study, the short aspect ratio of 2,430 lymph nodes was divided into three groups: <0.57; $0.57-0.81$ and $>0.81$, respectively. There is a significant difference of lymph node metastasis rates confirmed by pathology among three groups and $(P=0.005)$. In our study, the rate of positive lymph nodes in patients increased in the manner of the ratio of S/L depending. Thus, the S/L ratio of lymph nodes can be considered as a very important predictor of the presence or absence of lymph node metastases before operation.

The invasion of blood vessels and nerves by tumors has always been regarded as an important pathological factor. Some scholars have used it as one of the indicators of pathological grade. Theoretically, vascular invasion indicates that a large number of tumor cells enter the blood vessel and become the first step in tumor metastasis. Nerve encroachment can exist alone without vascular invasion and is another way of tumor spread and metastasis. However, there is still controversy about the relationship between vascular nerve invasion and cervical metastasis and prognosis. Some scholars have combined nerve invasion and vascular-lymphatic invasion as an independent factor in judging the early cervical cancer lymph node metastasis. Wu et al[34] divided 171 cases of early tongue cancer patients into two groups based on the presence or absence of nerve and vascular-lymphatic invasion, and found a significant difference in the rate of cervical lymph node metastases (presence or absence :44.4\% vs19.4\%) between groups. There is a higher neck recurrence rate in the patients with neurological and vascular-lymphatic-vessel invasion groups but without selective neck dissection in Wu's study. Multiple studies have shown that the presence of neural invasion predicts a poor prognosis of oral cancer [35-37]. Michikawa et al [38] reported 63 cases of tongue cancer patients with $50.8 \%$ of vascular invasion detected by immunohistochemistry, and found the close relationship between vascular invasion and tumor recurrence and prognosis. Chen TC et al [39] retrospectively studied 442 patients with early oral squamous cell carcinoma who underwent surgical treatment from 2004 to 2009 and investigated the effect of neurovascular infiltration on survival. They did not find the correlation between the neurovascular infiltration and 5-year disease-free survival and overall survival. Adel et al [40] 
retrospectively analyzed 571 patients with oral cancer, of which 5\% had lymphatic invasion and 3\% had vascular invasion. These two factors were found to have no significant correlation with local, neck recurrence and distant metastasis; in a multivariate analysis it cannot be used as an independent factor that affects the prognosis. Yeh et al [41] analyzed the correlation between various pathological factors and cervical metastases in 253 patients with early oral cancer. In patients without PNI or LVI (perineural invasion/lymph vascular invasion), the ultimate neck control rate $(96.9 \%$ vs. $96.3 \%, p=1.000)$ and 5-year disease-specific survival rate $(91.1 \%$ vs. $92.8 \%, \mathrm{p}=$ 0.863 ) were comparable between observation and END group. But there was a significantly higher incidence of neck recurrence found in observation group (Observation vs END: 16.9\%. 6.5\%, $\mathrm{p}=0.031$ ), with $93.8 \%$ occurring within one year. They recommended the observation under close follow-up for the first year for patients with in T1-2, cN0 OSCC without PNI or LVI. Our study found that 9 out of 161 patients with cN0 had peripheral vascular/nerve invasive patients. The probability of lymph node metastasis was not significantly increased compared with patients without peripheral vascular/ neurological invasion $(\mathrm{P}=0.27)$. Our results failed to show that the vascular/nerve invasion can be used as an important reference index for the management of cervical lymph nodes. The probably reason may be the insufficient number of patient with positive PNI/PVI included in the present study.

Here we found that clinical characterizes of patients such as gender, age, lesion location and $\mathrm{T}$ stage have no significant effect on cervical lymph node metastasis. Although the age is not an independent risk factor for cervical lymph node metastasis in oral squamous cell carcinoma patients with cN0, END surgical plans should be carefully selected for poor elderly patients.

We recognize that this study has its limitations. First, the research is a single center retrospective analysis. Second, the confirmation of cN0 before operation is just based on the regular physical examination and CT or MRI imaging scan, not every patient received PET-CT scan. The study would have been strengthened in the future.

\section{Conclusions}

Patients with oral squamous cell carcinoma undergoing neck lymph node dissection should thoroughly clean areas I, II, and III in order to maximize the removal of occult lymph node metastases. If the $\mathrm{cN} 0$ oral cancer patients have the independent risk factors such as invasive growth pattern, poor differentiation, high neutrophil/ lymphocyte rationed high short/long axis ratio of lymph node diameters, they should be given to the corresponding cervical lymph node dissection; if patients with the above risk factors receive nonstandard radical neck dissection or no dissection, it may be necessary for them to receive the corresponding regional postoperative radiotherapy.

\section{Acknowledgements}

\section{Ethics, consent and permissions}

All procedures performed in studies involving human participants were in accordance with the ethical standards of the institutional and/or national research committee and with the 1964 Helsinki declaration and its later amendments or comparable ethical standards. Our study was supported by the Ethics Committee of Zhongnan Hospital of Wuhan University. This article does not contain any studies with animals.

\section{Consent to publish}

We have obtained consent to publish from the participants to report individual patient data.

\section{Availability of data and material}

The datasets used and analysed during the current study are available from the corresponding author on reasonable request.

\section{Funding}

This research was partially funded by National Natural Science Foundation of China (No.81703872), Natural Science Foundation of Hubei province (No.2018CFB561) and Zhongnan Hospital of Wuhan University Science, Technology and Innovation Seed Fund (No. ZNPY2016043).

\section{Authors' contributions}

YL, KL, YK, YZ1 and MC performed all data collection and analysis, and $\mathrm{YL}$ drafted the manuscript. WL1, WL2, XH, ZL, YZ2 and CX provided professional advice on the experimental design and reviewed the paper. YZ1 corresponded to Yifei Zeng and WL1 corresponded to Wei Li. HY managed the experimental design, reviewed the manuscript and provided funding support. All the authors read and approved the final manuscript.

\section{Competing Interests}

The authors have declared that no competing interest exists.

\section{References}

1. Chaturvedi AK, Anderson WF, Lortet-Tieulent J, Curado MP, Ferlay J, Franceschi S, et al. Worldwide trends in incidence rates for oral cavity and 
oropharyngeal cancers. Journal of clinical oncology : official journal of the American Society of Clinical Oncology. 2013; 31: 4550-9.

2. Chen W, Zheng R, Baade PD, Zhang S, Zeng H, Bray F, et al. Cancer statistics in China, 2015. CA Cancer J Clin. 2016; 66: 115-32.

3. Siegel RL, Miller KD, Jemal A. Cancer Statistics, 2017. CA Cancer J Clin. 2017; 67: 7-30.

4. Bittar RF, Ferraro HP, Ribas MH, Lehn CN. Predictive factors of occult neck metastasis in patients with oral squamous cell carcinoma. Brazilian journal of otorhinolaryngology. 2016; 82: 543-7.

5. Kakuya T, Mori T, Yoshimoto S, Watabe Y, Miura N, Shoji H, et al. Prognostic significance of gene amplification of ACTN4 in stage I and II oral tongue cancer. Int J Oral Maxillofac Surg. 2017; 46: 968-76.

6. Pentenero M, Gandolfo S, Carrozzo M. Importance of tumor thickness and depth of invasion in nodal involvement and prognosis of oral squamous cell carcinoma: a review of the literature. Head Neck. 2005; 27: 1080-91.

7. Ganly I, Patel S, Shah J. Early stage squamous cell cancer of the oral tongue--clinicopathologic features affecting outcome. Cancer. 2012; 118: 101-11.

8. Castelijns JA, van den Brekel MW. Imaging of lymphadenopathy in the neck. Eur Radiol. 2002; 12: 727-38.

9. Forghani R, Yu E, Levental M, Som PM, Curtin HD. Imaging evaluation of lymphadenopathy and patterns of lymph node spread in head and neck cancer. Expert Rev Anticancer Ther. 2015; 15: 207-24.

10. Dogan E, Cetinayak HO, Sarioglu S, Erdag TK, Ikiz AO. Patterns of cervical lymph node metastases in oral tongue squamous cell carcinoma: implications for elective and therapeutic neck dissection. The Journal of laryngology and otology. 2014; 128: 268-73.

11. Bobdey S, Sathwara J, Jain A, Saoba S, Balasubramaniam G. Squamous cell carcinoma of buccal mucosa: An analysis of prognostic factors. South Asian J Cancer. 2018; 7: 49-54.

12. O'Donnell RK, Kupferman M, Wei SJ, Singhal S, Weber R, O'Malley B, et al. Gene expression signature predicts lymphatic metastasis in squamous cell carcinoma of the oral cavity. Oncogene. 2005; 24: 1244-51.

13. Jiang $\mathrm{CH}$, Jian $\mathrm{XC}$, Cheng $\mathrm{HQ}$. [Clinical analysis of cervical lymph node metastasis as primary manifestation of oral cancer]. Hunan Yi Ke Da Xue Xue Bao. 2003; 28: 657-8.

14. Akhter M, Hossain S, Rahman QB, Molla MR. A study on histological grading of oral squamous cell carcinoma and its co-relationship with regional metastasis. Journal of oral and maxillofacial pathology : JOMFP. 2011; 15: $168-76$.

15. Farhan-Alanie OM, McMahon J, McMillan DC. Systemic inflammatory response and survival in patients undergoing curative resection of oral squamous cell carcinoma. Br J Oral Maxillofac Surg. 2015; 53: 126-31.

16. Monroe MM, Gross ND. Evidence-based practice: management of the clinical node-negative neck in early-stage oral cavity squamous cell carcinoma. Otolaryngol Clin North Am. 2012; 45: 1181-93.

17. Koo BS, Choi EC, Yoon YH, Kim DH, Kim EH, Lim YC. Predictive factors for ipsilateral or contralateral central lymph node metastasis in unilateral papillary thyroid carcinoma. Ann Surg. 2009; 249: 840-4.

18. Lee CC, Lin YS, Kang BH, Chang KP, Chi CC, Lin MY, et al. Incorporation of log odds of positive lymph nodes into the AJCC TNM classification improves prediction of survival in oral cancer. Clinical otolaryngology : official journal of ENT-UK ; official journal of Netherlands Society for Oto-Rhino-Laryngology \& Cervico-Facial Surgery. 2017; 42: 425-32.

19. Olzowy B, Tsalemchuk Y, Schotten KJ, Reichel O, Harreus U. Frequency of bilateral cervical metastases in oropharyngeal squamous cell carcinoma: a retrospective analysis of 352 cases after bilateral neck dissection. Head \& neck. 2011; 33: 239-43.

20. Woolgar JA. The topography of cervical lymph node metastases revisited: the histological findings in 526 sides of neck dissection from 439 previously untreated patients. International journal of oral and maxillofacial surgery. 2007; 36: 219-25.

21. Yuen AP, Lam KY, Wei WI, Lam KY, Ho CM, Chow TL, et al. A comparison of the prognostic significance of tumor diameter, length, width, thickness, area, volume, and clinicopathological features of oral tongue carcinoma. Am J Surg. 2000; 180: 139-43.

22. Kane SV, Gupta M, Kakade AC, A DC. Depth of invasion is the most significant histological predictor of subclinical cervical lymph node metastasis in early squamous carcinomas of the oral cavity. Eur J Surg Oncol. 2006; 32: 795-803.

23. Fukano H, Matsuura H, Hasegawa Y, Nakamura S. Depth of invasion as a predictive factor for cervical lymph node metastasis in tongue carcinoma. Head Neck. 1997; 19: 205-10.

24. Melchers LJ, Schuuring E, van Dijk BA, de Bock GH, Witjes MJ, van der Laan $\mathrm{BF}$, et al. Tumour infiltration depth $>/=4 \mathrm{~mm}$ is an indication for an elective neck dissection in pT1cN0 oral squamous cell carcinoma. Oral Oncol. 2012; 48: $337-42$

25. Mishra N, Rath KC, Upadhyay UN, Raut S, Baig SA, Birmiwal KG. Preoperative evaluation of cervical lymph nodes for metastasis in patients with oral squamous cell carcinoma: A comparative study of efficacy of palpation, ultrasonography and computed tomography. National journal of maxillofacial surgery. 2016; 7: 186-90.

26. Haksever M, Inancli HM, Tuncel U, Kurkcuoglu SS, Uyar M, Genc O, et al. The effects of tumor size, degree of differentiation, and depth of invasion on the risk of neck node metastasis in squamous cell carcinoma of the oral cavity. Ear Nose Throat J. 2012; 91: 130-5

27. Alves AM, Diel LF, Lamers ML. Macrophages and prognosis of oral squamous cell carcinoma: A systematic review. J Oral Pathol Med. 2018; 47: 460-7.

28. Bowen RC, Little NAB, Harmer JR, Ma J, Mirabelli LG, Roller KD, et al. Neutrophil-to-lymphocyte ratio as prognostic indicator in gastrointestinal cancers: a systematic review and meta-analysis. Oncotarget. 2017; 8: 32171-89.

29. Nakashima H, Matsuoka Y, Yoshida R, Nagata M, Hirosue A, Kawahara K, et al. Pre-treatment neutrophil to lymphocyte ratio predicts the chemoradiotherapy outcome and survival in patients with oral squamous cell carcinoma: a retrospective study. BMC Cancer. 2016; 16: 41.

30. Bobdey S, Ganesh B, Mishra P, Jain A. Role of Monocyte Count and Neutrophil-to-Lymphocyte Ratio in Survival of Oral Cancer Patients. Int Arch Otorhinolaryngol. 2017; 21: 21-7.

31. Steinkamp HJ, Cornehl M, Hosten N, Pegios W, Vogl T, Felix R. Cervical lymphadenopathy: ratio of long- to short-axis diameter as a predictor of malignancy. Br J Radiol. 1995; 68: 266-70.

32. Lin CM, Wang CP, Chen CN, Lin CY, Li TY, Chou CH, et al. The application of ultrasound in detecting lymph nodal recurrence in the treated neck of head and neck cancer patients. Sci Rep. 2017; 7: 3958.

33. Song WS, Yang YP, Huang CS, Lu KH, Liu WH, Wu WW, et al. Sox2, a stemness gene, regulates tumor-initiating and drug-resistant properties in CD133-positive glioblastoma stem cells. Journal of the Chinese Medical Association : JCMA. 2016; 79: 538-45.

34. Wu K, Yang X, Li L, Ruan M, Liu W, Lu W, et al. Neurovascular Invasion and Histological Grade Serve as the Risk Factors of Cervical Lymph Node Metastases in Early Tongue Squamous Cell Carcinoma. Mol Neurobiol. 2016; 53: 2920-6.

35. Tai SK, Li WY, Yang MH, Chang SY, Chu PY, Tsai TL, et al. Treatment for T1-2 oral squamous cell carcinoma with or without perineural invasion: neck dissection and postoperative adjuvant therapy. Ann Surg Oncol. 2012; 19: 1995-2002

36. Aivazian K, Ebrahimi A, Low TH, Gao K, Clifford A, Shannon K, et al. Perineural invasion in oral squamous cell carcinoma: quantitative subcategorisation of perineural invasion and prognostication. J Surg Oncol. 2015; 111: 352-8

37. Chinn SB, Spector ME, Bellile EL, McHugh JB, Gernon TJ, Bradford CR, et al. Impact of perineural invasion in the pathologically N0 neck in oral cavity squamous cell carcinoma. Otolaryngol Head Neck Surg. 2013; 149: 893-9.

38. Michikawa C, Uzawa N, Kayamori K, Sonoda I, Ohyama Y, Okada N, et al. Clinical significance of lymphatic and blood vessel invasion in oral tongue squamous cell carcinomas. Oral Oncol. 2012; 48: 320-4.

39. Chen TC, Wang CP, Ko JY, Yang TL, Hsu CW, Yeh KA, et al. The impact of perineural invasion and/or lymphovascular invasion on the survival of early-stage oral squamous cell carcinoma patients. Ann Surg Oncol. 2013; 20: 2388-95.

40. Adel M, Kao HK, Hsu CL, Huang JJ, Lee LY, Huang Y, et al. Evaluation of Lymphatic and Vascular Invasion in Relation to Clinicopathological Factors and Treatment Outcome in Oral Cavity Squamous Cell Carcinoma. Medicine (Baltimore). 2015; 94: e1510

41. Yeh CF, Li WY, Yang MH, Chu PY, Lu YT, Wang YF, et al. Neck observation is appropriate in T1-2, $\mathrm{cN0}$ oral squamous cell carcinoma without perineural invasion or lymphovascular invasion. Oral Oncol. 2014; 50: 857-62. 